\title{
Prefrontal glucose metabolism in medication-resistant major depression
}

\author{
Cheng-Ta Li, Tung-Ping Su, Shyh-Jen Wang, Pei-Chi Tu and Jen-Chuen Hsieh
}

\section{Background}

Medication-resistant depression (MRD) is associated with poorer attentional performance and immense socioeconomic costs.

\section{Aims}

We aimed to investigate the central pathophysiology of MRD, previously linked to impaired prefrontal cortex function.

\section{Method}

A total of 54 participants (22 with MRD, 16 with non-resistant depression, 16 healthy controls) were recruited. Non-MRD status was confirmed by a prospective 6-week antidepressant trial. All medication-free participants underwent a go/no-go task to study prefrontal cortical function (attention) and positron emission tomography scans to study regional cerebral glucose metabolism (rCMglu) at rest.

\section{Results}

The MRD group had worse attentional ratings and decreased rCMglu compared with the non-MRD and control groups. Attentional performance was positively associated with prefrontal cortex rCMglu. The prefrontal cortex differences between MRD and non-MRD groups remained after adjusting for past depressive episodes $\left(F_{(1,35)}=4.154\right.$, $P=0.043$ ).

\section{Conclusions}

Pronounced hypofrontality, with the associated attentional deficits, has a key role in the neuropathology of medicationresistant depression.

\section{Declaration of interest}

None.
A substantial proportion of people with major depressive disorder fail to respond to sequential trials of antidepressant treatments. Such treatment-resistant depression may account for the immense socioeconomic burden caused by the disorder. Unresolved symptoms of depression are associated with greater cognitive deficits (e.g. in attentional performance), ${ }^{2}$ lower psychosocial functioning and increased suicidality. ${ }^{3}$ The conceptual and operational definitions of treatment-resistant depression vary across studies. ${ }^{4}$ Even so, major depression is usually considered as resistant or refractory when at least two adequate trials with antidepressants from different pharmacologic classes fail to achieve clinical remission, ${ }^{5,6}$ regardless of other treatment options (e.g. psychotherapy and brain stimulation) and other characteristics of treatment-resistant depression (e.g. severity and duration). Many psychosocial factors are related to treatment resistance. However, there is limited evidence thus far supporting the effectiveness of psychotherapy for medication-resistant depression (MRD). ${ }^{5}$ For example, patients with citalopram-resistant depression receiving cognitive-behavioural therapy in combination with medication had responses and remission rates similar to those of patients taking medications alone. ${ }^{7}$ In fact, MRD is not uncommon in patients without prominent psychosocial stressors. Brain functional abnormalities have a significant role in the pathophysiology of major depressive disorder. ${ }^{8}$ A recent review of the neuroimaging evidence on treatment effects in depression indicated that not all dysfunctional processes associated with depression can be reversed by antidepressant treatment and that trait characteristics are evident on both behavioural and neurobiological levels. ${ }^{9}$ Although major depression is considered heterogeneous and treatment outcome might be affected by individual psychosocial factors, the worsened cognitive function that differentiates MRD from non-MRD suggests that the former condition might have some common neurobiological features across cases. However, the central pathophysiology that specifically underpins medication resistance remains elusive.
The prefrontal cortex is a key neural substrate in the corticolimbic network and the dysfunction of this network is thought to be a core deficit in depression. ${ }^{10}$ The prefrontal cortex could possibly exert top-down control over limbic hyperactivity as a regulatory mechanism for depression, or could reduce negative emotions. ${ }^{11}$ The enhancement of this top-down regulatory mechanism, e.g. by repetitive transcranial magnetic stimulation of the dorsolateral prefrontal cortex, ${ }^{12}$ has been shown to substantially improve depression that had responded poorly to antidepressant medications. Decreased prefrontal cortex glucose metabolism was reported to be significantly correlated with depression severity (as measured by rating scales) in people with major depressive disorder. ${ }^{13}$ However, it remains elusive whether the prefrontal cortex hypometabolism reflected a state-dependent characteristic (e.g. depression rating scores in the past week) or a trait-dependent characteristic (i.e. antidepressant refractoriness) of depressive severity. We predicted that decreased prefrontal cortex glucose metabolism would be a trait feature of patients with MRD, but not of those amenable to treatment. Indirect evidence supported that the former patients may have more prominent hypofrontality than those without treatment resistance. $^{14}$

Although demographic variables and state-dependent depressive severity, as rated by objective rating scales, might not distinguish between patients with and without a prominent history of antidepressant resistance, we aimed to investigate the central pathophysiology of MRD. We specifically predicted that decreased prefrontal cortex glucose metabolism would be the neuroimaging hallmark that differentiates MRD and non-MRD endophenotypes. Using positron emission tomography (PET), brain $\left[{ }^{18} \mathrm{~F}\right]$ fluoro-2-deoxy-D-glucose (FDG) uptake is indicative of regional cerebral glucose metabolism (rCMglu) at rest. ${ }^{15} \mathrm{~A}$ visual 'go/no-go' task to assess a prefrontal cortical-related function, such as attention, was implemented as a behavioural probe for the cognitive deficits associated with depression. ${ }^{2}$ We also aimed to elucidate whether altered glucose metabolism was 
correlated with clinical depressive severity and the go/no-go task performance indices in MRD and non-MRD patient groups having comparable demographic features and state-dependent depressive severity.

\section{Method}

Patients with major depressive disorder were recruited for this study from September 2010 to January 2012. The diagnoses were established by structural history-taking and administration of the Mini International Neuropsychiatric Interview (MINI) based on DSM-IV criteria. ${ }^{16,17}$ Adult patients (age range 21-65 years) were recruited. Exclusion criteria included major physical or neurological disorder, a history of alcohol or substance misuse or dependence, and major psychiatric comorbidities such as schizophrenia, bipolar disorder, other major psychosis, obsessive-compulsive disorders, post-traumatic stress disorder or Cluster B personality disorders. The DSM-IV diagnostic criteria for personality disorders were adopted. ${ }^{17}$

Three groups of participants were finally recruited for this analysis: an MRD group $(n=22)$, a non-MRD group $(n=16)$ and a healthy control group $(n=16)$ (see Fig. 1). We adopted the most widely accepted criteria of a clear history of MRD (a failure to respond to at least two different classes of adequate antidepressant trials in the present episode or in the past) for the recruitment of the MRD group. ${ }^{5,6}$ These participants also needed to have a history of failure to respond to one type of selective serotonin reuptake inhibitor (SSRI). Participants in the non-MRD group had no history of medication resistance and responded well to antidepressants as confirmed by a prospective 6-week adequate trial with an SSRI (sertraline, citalopram or escitalopram) (see online supplement DS1 for details). The control group comprised healthy individuals well matched for age, gender, education and handedness. Furthermore, in the 6-week SSRI treatment trial, patients who did not have more than a $50 \%$ improvement in depression were considered as intermediate level to treat, and thus not a primary study group (the data from this group are also reported; see Results section). The study was performed in accordance with the Declaration of Helsinki and was approved by the appropriate local ethics review committee. All participants provided written informed consent.

\section{Study procedures}

Baseline medication-free PET scans, psychiatric ratings and cognitive testing were performed on the same day for each participant. Demographic and clinical characteristics were obtained for all participants based on a structural interview and a thorough recording of their medical histories. Individual assessments of mood severity were performed by an experienced psychiatrist and included ratings from the 17-item Hamilton Rating Scale for Depression (HRSD-17) and the Young Mania Rating Scale (YMRS). ${ }^{18,19}$ Neurocognitive tests (see below) were performed by a trained research assistant. For ethical reasons the non-MRD group comprised patients who were newly diagnosed or relapsed and had been without medications for at least 2 weeks before their recruitment. Structural magnetic resonance imaging (MRI) imaging scans were acquired on the same day as the other tests or (when clinical limitations were present) within at most 5 days of the study day.

\section{Neurocognitive tests for attentional performance}

The go/no-go task, used in our earlier study, ${ }^{2}$ was adopted to test prefrontal cortical-related function such as attentional performance. It is computer-based and all the tasks were generated on a computer screen ( $32 \mathrm{~cm}$ diagonal screen size) with a viewing distance of $60 \mathrm{~cm}$. Participants were asked to respond as quickly as possible after the ' $x$ ' symbol appeared and not to press the key when the ' + ' symbol appeared. During the testing process participants were seated in a comfortable chair with arm rests. They were asked to press a key in the front central area of the keyboard with their right index finger as quickly as possible, according to the given testing instructions. Pretest trials were provided to ensure that participants understood each task. After they had completed the pretest with all correct responses, the formal test was then administered. The reaction times (mean, median and standard deviations) and omission frequencies were recorded as index measurements for attentional performance.

\section{Imaging procedure}

Magnetic resonance imaging $T_{1}$-weighted images were acquired (mainly to improve spatial registration and normalisation of PET data) on a 3 T Discovery 750 whole-body high-speed imaging device (GE Healthcare, www.gehealthcare.com). Resting-state PET scans of glucose utilisation were acquired on a Discovery VCT PET/CT scanner (GE Healthcare) with a three-dimensional (3D) brain mode. Most of the PET scans were done at around noon $(11.00 \mathrm{~h}$ to $14.00 \mathrm{~h})$; participants fasted for at least $4 \mathrm{~h}$ beforehand. The PET images were acquired in the $45 \mathrm{~min}$ after an intravenous injection of approximately $370 \mathrm{MBq}$ of $\left[{ }^{18} \mathrm{~F}\right] \mathrm{FDG}$. The brain acquisition time was $15 \mathrm{~min}$. After the $\left[{ }^{18} \mathrm{~F}\right] \mathrm{FDG}$ injection, participants were instructed not to do anything except wait in a quiet room with their eyes open (see online supplement DS1 for more details).

\section{Processing of PET data}

All pre-processing and image analyses were performed using Statistical Parametric Mapping software (SPM8) for Windows (http://www. fil.ion.ucl.ac.uk/spm). A group-specific template of images was first established directly from the data-sets of the participants to reduce any bias in template selection and improve accuracy in PET spatial normalisation. The anatomical MRI and PET scans of each individual were co-registered and then MRI images were normalised to the standard MRI $T_{1}$ template. The resulting deformation field obtained from the latter procedure was applied to the PET scans. Finally, these normalised PET images from all participants were averaged to create a groupspecific, MRI-aided $\left[{ }^{18} \mathrm{~F}\right] \mathrm{FDG}$ template. ${ }^{20}$ Spatial normalisation by such a template provides the highest spatial specificity and sensitivity compared with use of other standard templates. ${ }^{20}$ Each participant's images were normalised to the study-specific $\left[{ }^{18} \mathrm{~F}\right]$ FDG template. Normalised PET images were further smoothed with an isotropic $10 \mathrm{~mm}$ 3D Gaussian kernel before voxel-wise analysis.

\section{Statistical analysis}

For analysis of demographic data and clinical variables SPSS version 16.0 software was used. One-way analyses of variance (ANOVAs) (or Student's $t$-tests) and chi-squared tests were applied to compare the continuous and categorical variables respectively among groups. The significance level was set at $P<0.05$.

\section{Voxel-wise analysis of PET data}

The overall grand mean of the PET scans was centred and normalised to $100 \mu \mathrm{mol} / 100 \mathrm{ml}$ per minute, and global variance across scans was removed by analysis of covariance (ANCOVA). 
To assess potential group differences of normalised brain glucose uptakes, an ANCOVA with age, gender and global grey-matter values as covariates of no interest was used for between-group comparisons, and the main effects are reported (MRD $v$. non-MRD $v$. control). The significance thresholds were set at $P<0.05$ at the voxel level, and family-wise error (FWE) was applied to correct for multiple comparisons. For the post hoc analyses, independent $t$-tests were performed for the between-group comparisons (MRD v. non-MRD, MRD $v$. controls, non-MRD $v$. controls) within the identified brain regions that passed voxel-level FWE-corrected $P<0.05$ in the three-group comparison. The statistical thresholds were set at $P<0.0167$ ( $=0.05 / 3$, corrected for the three groups). Voxel-based partial correlations were performed to investigate the association between past major depressive episodes (and illness duration) and $\left[{ }^{18} \mathrm{~F}\right]$ FDG uptake after controlling for age, gender, HRSD-17 score and total grey-matter counts. The significance thresholds were also set at a voxel-level FWE-corrected $P<0.05$.

\section{Region of interest analysis}

Mean brain glucose uptake values in the prefrontal cortex were extracted from unsmoothed PET images in the standard stereotactic space by using PMOD version 3.0 (PMOD Technologies, Zurich, Switzerland) as previously described. ${ }^{21}$ Normalised prefrontal cortex glucose uptakes (values were normalised by dividing the prefrontal cortex uptake values by the global mean uptake values) were reported. In brief, an automated anatomical labelling template was used ${ }^{22}$ to help delineate the prefrontal cortex to prevent bias from inter- or intrarater reliability due to manual delineation. The delineated prefrontal cortex covered the clusters that are most involved in depression, including the dorsolateral prefrontal cortex. ${ }^{23}$ Correlation tests were performed to study the correlations between depression scores, attentional measurements and normalised metabolic activities in the prefrontal cortex, without (by Pearson's correlation tests) and with (by partial correlation tests) past major depressive episodes as the covariate of no interest. One-way ANOVA tests were performed on the normalised prefrontal cortex rCMglu values across the three groups, and these were followed by post hoc least significant difference analyses. A $P$ value of less than 0.05 for the correlation analyses and post hoc analyses was deemed to be statistically significant. Receiver operating characteristic (ROC) curves were plotted for assessing the accuracy of prediction of antidepressant outcome by normalised glucose uptake. The area under the ROC curve (AUC) and the optimal cut-off based on highest sensitivity and specificity were calculated. ${ }^{24}$ Finally, multivariate logistic regression analysis was carried out, with age, gender, HRSD-17 score, past depressive episodes, duration of illness and normalised prefrontal cortex rCMglu treated as independent factors and a diagnosis of MRD as the dependent factor. The adjusted odds ratios of MRD risk and their 95\% confidence intervals were reported. Statistical significance was assigned to differences having $P<0.05$ (two-sided tests).

\section{Results}

Sample recruitment is summarised in Fig. 1. Demographic data, mood ratings and illness characteristics did not differ among

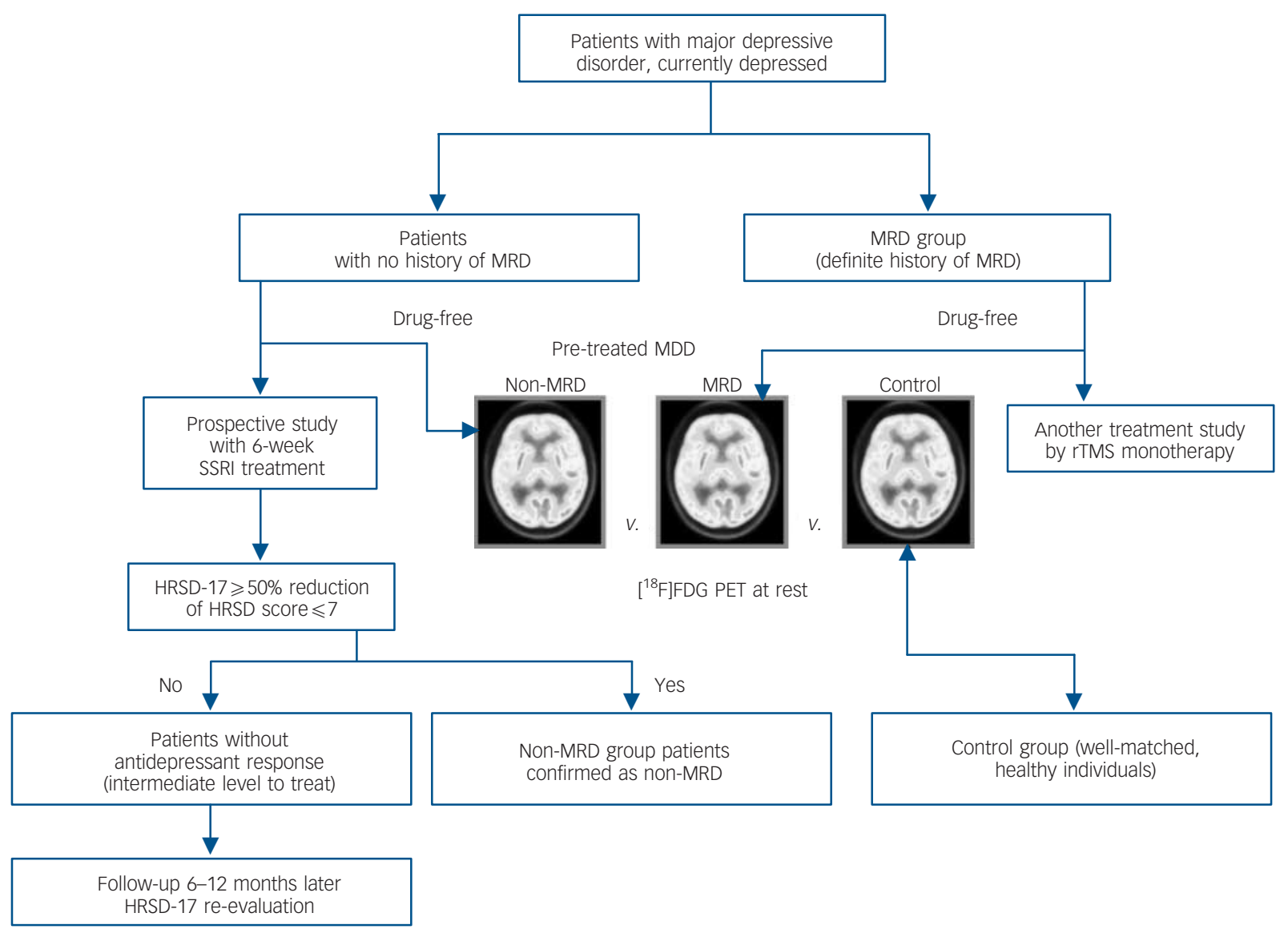

Fig. 1 Study profile. HRSD, Hamilton Rating Scale for Depression; MDD, major depressive disorder; MRD, medication-resistant depression; PET, positron emission tomography; rTMS, repetitive transcranial magnetic stimulation; SSRI, serotonic-specific reuptake inhibitor. 
the three groups, except that the patients in the MRD group had greater numbers of past major depressive episodes than the nonMRD group (mean episodes MRD 4.4, s.d. =3.0, v. non-MRD 2.5, s.d. $\left.=1.1 ; \quad F_{(2,51)}=6.90, P<0.05\right) \quad$ (Table 1). All the patients recruited to the MRD group had a history of failure to respond to augmentation treatment with medication combinations or add-on psychotherapeutic interventions. All the patients recruited to the non-MRD group improved significantly after 6 weeks of SSRI treatment (post-treatment HRSD-17 score 3.2, s.d.=2.8). No difference in psychiatric comorbidity was noted between the MRD and non-MRD groups: panic disorder 23\% (MRD) $v$. 19\% (non-MRD); social phobia 4\% (MRD) v. 6\% (non-MRD); generalised anxiety disorder $41 \%$ (MRD) v. 44\% (non-MRD); all $P>0.10$. The MRD and non-MRD groups did not differ in terms of the length of the current episode (MRD 5.6 months, s.d. $=3.7, v$. non-MRD 9.5 months, s.d. $=9.6, t=1.703, P=0.112$ ). A greater tendency to omit visual stimuli in the environment (omissions, $F_{(2,51)}=3.398, P<0.05$ ) was observed in the MRD group compared with the non-MRD and control groups. Moreover, subjective psychosocial stressors were reported in $21 \%(n=8)$ of all patients, but there was no significant difference between the MRD group $(23 \%, n=5)$ and the non-MRD groups $(19 \%$, $n=3$ ). Such results suggested that treatment refractoriness might not be simply explained by the existence of psychosocial factors.

\section{Between-group differences in rCMglu}

\section{Voxel-wise analysis}

Significant rCMglu differences in the bilateral dorsolateral regions of the prefrontal cortex (Brodmann's area 9), supplementary motor area (BA 6) and left middle temporal gyrus were noted in the three-group comparisons (Fig. 2a); see also online Table DS1. The post hoc analysis showed that rCMglu in the bilateral dorsolateral prefrontal cortex and supplementary motor area was decreased in the MRD group compared with the non-MRD group (Fig. 2b). Compared with the control group, both MRD and non-MRD groups demonstrated decreased rCMglu in the left middle and inferior temporal gyri (BA 20 and 21); however, the MRD group patients also had decreased rCMglu in the bilateral dorsolateral prefrontal cortex and supplementary motor area (see online Fig. DS1).

\section{Region of interest analysis}

We found that the decrease in prefrontal cortex rCMglu was significantly more prominent in the MRD group compared with the non-MRD and control groups $\left(F_{(2,51)}=11.071, P<0.001\right.$; Fig. 2c). The correlation between prefrontal cortex rCMglu and past major depressive episodes $(r=-0.300, P=0.067)$ was not statistically significant. After controlling out the numbers of past major depressive episodes, the differences in prefrontal cortex rCMglu still existed between the MRD and non-MRD groups $\left(F_{(1,35)}=4.154, P=0.043\right)$. We further analysed rCMglu among groups in some brain areas that were not a priori regions of interest, including subregions of the prefrontal cortex, anterior cingulate cortex and amygdala (online Fig. DS2). The most significant finding was in the bilateral dorsolateral prefrontal cortex, which was consistent with the findings of the voxel-wise analysis. Furthermore, 11 patients with major depressive disorder could not be categorised as non-MRD or MRD after the 6-week SSRI treatment. Despite the fact that it was not a primary study purpose, we found that these intermediate level to treat patients had no prominent prefrontal cortex rCMglu decrease (online Fig. DS3). In the post-study follow-up period (range 6-12 months) we found that most of these patients were eventually stabilised by a longer period of SSRI treatment $(n=3)$ or by switching to a different class of antidepressant $(n=4)$. These results suggested that prefrontal cortical rCMglu is not a good predictor of response to short-term antidepressant treatment but that it is a characteristic feature for a subgroup of patients with depression resistant to antidepressant medication.

\section{Attentional ratings, MDEs and rCMglu}

Faster mean reaction times were significantly correlated with higher rCMglu in the prefrontal cortex $(r=-0.449, P<0.005)$ (Fig. 2d) and the negative correlation remained significant even after controlling out past depressive episodes in the statistical model (Table 2). No significant correlation was found between omission and standard deviation of the reaction times and the prefrontal cortex rCMglu. Individual testing of association in each of the groups also revealed a similar trend that faster mean reaction times were significantly correlated with higher rCMglu in the prefrontal cortex (online Table DS2). We also found no

Table 1 Demographic variables, illness characteristics and attentional performance

\begin{tabular}{|c|c|c|c|c|c|}
\hline & MRD group & Non-MRD group & Control group & $F / t / \chi^{2}$ & $P$ \\
\hline \multicolumn{6}{|l|}{ Demographic variables } \\
\hline Gender: female, \% & 50 & 50 & 50 & & \\
\hline Right-handedness, \% & 100 & 100 & 100 & & \\
\hline Age, years: mean (s.d.) & $41.5(13.0)$ & $43.5(10.4)$ & $42.0(10.2)$ & $0.813(F)$ & 0.492 \\
\hline Education, years: mean (s.d.) & $12.2(3.9)$ & $13.3(2.8)$ & $13.2(2.9)$ & $0.654(F)$ & 0.584 \\
\hline \multicolumn{6}{|l|}{ IIIness characteristics } \\
\hline Duration, years: mean (s.d.) & $12.3(4.0)$ & $13.3(2.6)$ & & $0.816(t)$ & 0.420 \\
\hline Number of MDE: mean (s.d.) & $4.4(3.0)$ & $2.5(1.1)$ & & $-2.770(t)$ & 0.026 * \\
\hline HRSD-17 score: mean (s.d.) & $20.3(5.9)$ & $20.1(5.7)$ & & $-0.083(t)$ & 0.934 \\
\hline YMRS: mean (s.d.) & $1.1(1.6)$ & $1.7(1.7)$ & & $1.187(t)$ & 0.243 \\
\hline Suicidal history, n (\%) & $6(27.0)$ & $2(12.5)$ & & $0.426\left(\chi^{2}\right)$ & 0.245 \\
\hline Psychotic features, n (\%) & $4(18.2)$ & $1(6.3)$ & & $0.374\left(\chi^{2}\right)$ & 0.286 \\
\hline \multicolumn{6}{|l|}{ Attention (go/no-go task) ${ }^{a}$} \\
\hline \multicolumn{6}{|l|}{ Reaction time, ms } \\
\hline Mean (s.d.) & $520.2(111.7)^{b}$ & 467.1 (64.3) & $448.4(69.7)$ & $3.219(F)$ & $0.048^{*}$ \\
\hline Median (s.d.) & $498.2(107.2)$ & 460.8 (54.5) & 454.7 (71.6) & $1.577(F)$ & 0.217 \\
\hline s.d. (s.d.) & $85.2(36.4)^{b}$ & 74.9 (15.6) & $63.3(16.3)$ & $3.184(F)$ & $0.049 *$ \\
\hline No. of omissions & $1.0(2.0)^{\mathrm{b}, \mathrm{c}}$ & $0.0(0.0)$ & $0.1(0.3)$ & $3.398(F)$ & $0.041^{*}$ \\
\hline \multicolumn{6}{|c|}{$\begin{array}{l}\text { HRSD-17, } 17 \text {-item Hamilton Rating Scale for Depression; MDE, major depressive episodes; MRD, medication-resistant depression; YMRS, Young Mania Rating Scale. } \\
\text { a. ANOVA, F(d.f.2,51) } \\
\text { b. POSt hoc MRD }>\text { control. } \\
\text { C. POSt hoc MRD > non-MRD. } \\
{ }^{*} P<0.05 \text {. }\end{array}$} \\
\hline
\end{tabular}



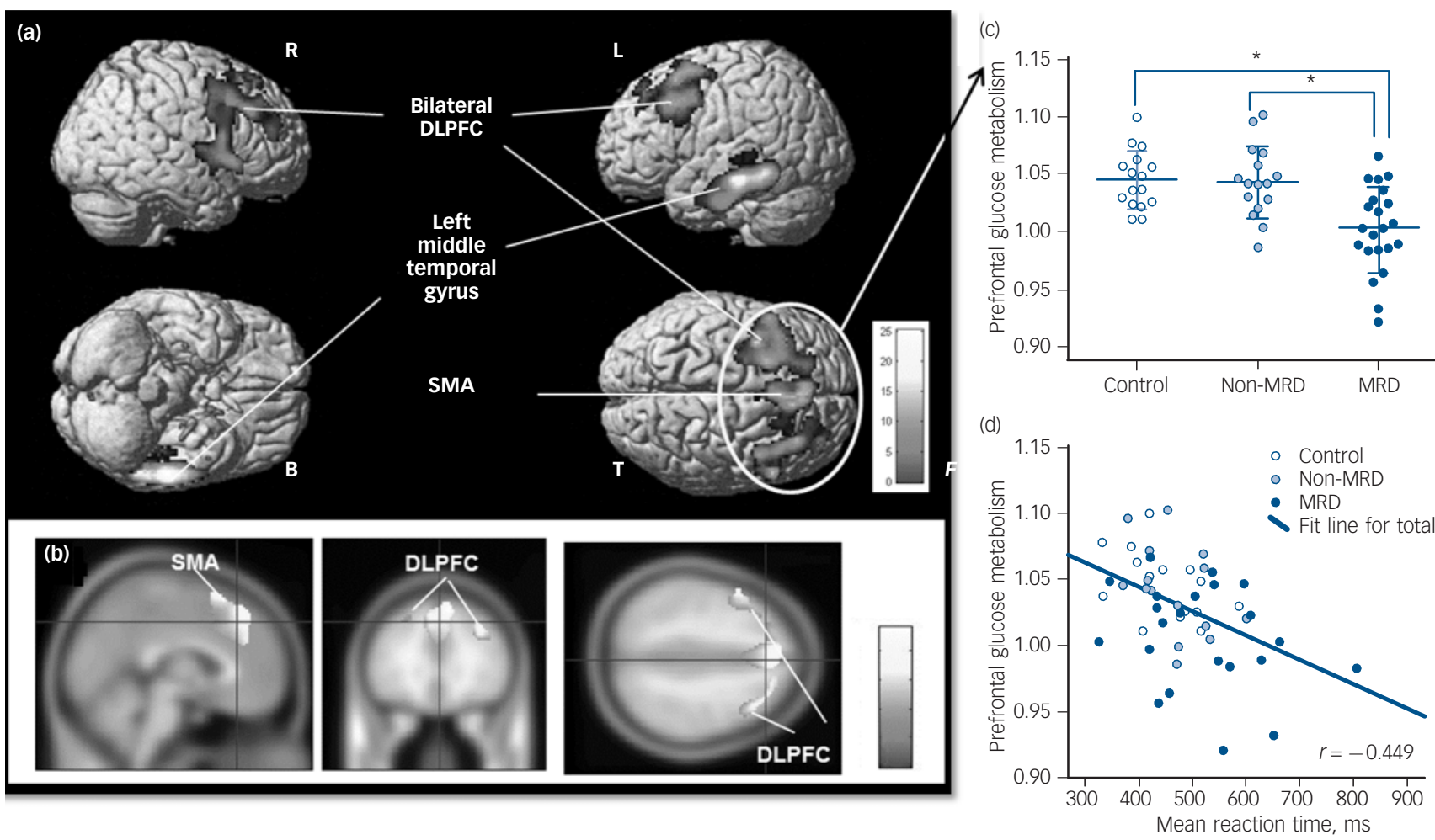

Fig. 2 Regional cerebral glucose metabolism (rCMglu) in the prefrontal cortices of patients with medication-resistant depression (MRD) compared with the non-MRD patient and control groups. (a) MRD v. non-MRD v. control: the whole-brain analytic results from analysis of covariance (ANCOVA) showed significant rCMglu differences in the bilateral dorsolateral prefrontal cortex (DLPFC), left middle temporal gyrus and supplementary motor area (SMA); thresholded at the voxel-level family-wise error (FWE)-corrected $P$ value of 0.05 , after adjusting for age, gender and global glucose uptakes. Bar denotes $F$ values; B, bottom view, L, left, R, right, T, top view. (b) MRD v. non-MRD: post hoc analysis showed that the MRD group had significantly lower rCMglu in the bilateral DLPFC and SMA. Bar denotes $t$ values. (c) Post hoc least significant difference analysis showed that the normalised prefrontal cortical rCMglu in the MRD group was significantly lower than in the non-MRD group (mean difference -0.041 ) and the control group (mean difference -0.0432 ). These differences still existed after controlling out the number of past major depressive episodes $\left(F_{(1,35)}=4.154, P=0.043\right)$. (d) Lower rCMglu in the prefrontal cortex was correlated with slower mean reaction times on the go/no-go attention task, explaining why the MRD group performed worse on this task than the other two groups; ${ }^{*} P<0.005$.

correlation between past depressive episodes and brain rCMglu; this was also true after lowering the statistical threshold to an uncorrected $P<0.005$.

\section{Predicting refractoriness by frontal rCMglu}

The AUC for prefrontal cortex glucose uptakes was 0.764 (online Fig. DS4). If we thresholded at 1.029 (normalised prefrontal cortex rCMglu, range $0.921-1.102$ ) based on highest sensitivity and specificity, the sensitivity and specificity to accurately predict the refractoriness group are $68 \%$ and $71 \%$, respectively. Regarding the multivariate logistic regression with age, gender, HRSD-17 score, past major depressive episodes, duration of illness and normalised prefrontal cortex rCMglu treated as independent variables, the variable most strongly associated with a diagnosis of MRD was prefrontal cortex rCMglu $(\beta=-51.01$, Wald $=4.98$,
$P=0.03$ ) (Table 3), indicating a strong negative correlation between prefrontal cortical rCMglu and the existence of a history of treatment refractoriness. These results suggest that the normalised prefrontal cortical rCMglu (i.e. prefrontal cortex relative to whole-brain glucose uptakes) is a characteristic feature of MRD, and thus could assist in differentiating patients with resistant disorder from the population with major depressive disorder.

\section{Discussion}

Patients with depression with and without a medication-resistant history could be differentiated by features related to resting brain glucose metabolism in the prefrontal cortex (i.e. dorsolateral prefrontal cortex and supplementary motor area), even when

\begin{tabular}{|c|c|c|c|c|}
\hline \multirow[b]{2}{*}{ PFC rCMglu } & \multicolumn{4}{|c|}{ Correlation coefficients } \\
\hline & Median $^{a}$ & Omission & s.d. ${ }^{a}$ & Mean \\
\hline $\begin{array}{l}\text { Pearson's correlation test } \\
\text { Partial correlation test controlling for MDES }\end{array}$ & $\begin{array}{l}-0.413^{* *}(P=0.002) \\
-0.310(P=0.066)\end{array}$ & $\begin{array}{l}-0.137(P=0.329) \\
-0.015(P=0.931)\end{array}$ & $\begin{array}{l}-0.260(P=0.060) \\
-0.144(P=0.401)\end{array}$ & $\begin{array}{l}-0.449 * *(P=0.001) \\
-0.342 *(P=0.041)\end{array}$ \\
\hline $\begin{array}{l}\text { MDE, major depressive episode; PFC, prefrontal co } \\
\text { a. Reaction time. } \\
{ }^{*} P<0.05,{ }^{*} P<0.01 \text {. }\end{array}$ & ג, regional cerebral gluco & & & \\
\hline
\end{tabular}




\begin{tabular}{|c|c|c|c|c|}
\hline Independent variables & $\beta$ & s.e. & OR (95\% Cl) & $P$ \\
\hline Age & -0.177 & 0.179 & $0.838(0.718-1.077)$ & 0.094 \\
\hline Gender & 0.516 & 1.219 & $0.672(0.154-18.262)$ & 0.672 \\
\hline HRSD-17 score & 0.162 & 0.099 & $1.175(0.969-1.426)$ & 0.102 \\
\hline Past MDEs & 0.773 & 0.438 & $2.165(0.918-5.105)$ & 0.077 \\
\hline Duration of illness & -0.154 & 0.255 & $0.858(0.520-1.415)$ & 0.547 \\
\hline PFC rCMglu & -51.012 & 22.859 & $0.000(0.000-0.002)$ & $0.026^{*}$ \\
\hline
\end{tabular}

these two groups were comparable in terms of demographic features, duration of illness and HRSD-17 scores. Prefrontal cortical rCMglu in the depressed status had acceptable, but not optimal, sensitivity and specificity as a biomarker for predicting a diagnosis of MRD. Treatment resistance cannot be explained simply by current psychosocial factors or past depressive episodes, which further supports the essential role of biological factors in MRD. There have been many previous studies using $\left[{ }^{18} \mathrm{~F}\right] \mathrm{FDG}$ PET in depression, yet the results so far are inconsistent. ${ }^{25-27}$ For example, both increased and decreased prefrontal cortex activities have been reported in depression. Our findings at least partly explain such inconsistency. Previous studies of patients with lower treatment refractoriness, such as symptomatic remission after medication treatment, ${ }^{25,26}$ or good response to cognitive-behavioural psychotherapy, ${ }^{25}$ did not tend to reveal prefrontal cortex hypometabolism, whereas patients having higher treatment refractoriness, such as those subjected to brain stimulation, were reported to have prefrontal cortex hypometabolism before treatment. ${ }^{27}$

Prefrontal cortex glucose metabolism in the MRD group was significantly decreased, as predicted, suggesting that a more functionally impaired prefrontal cortex could be one biological factor in the observed treatment refractoriness of major depression. The finding of hypofrontality in the MRD group could be one key to their preponderant cognitive functional problems, as demonstrated by the correlation of hypofrontality with attentional deficits (see Fig. 2d). We also found that the supplementary motor area glucose metabolism in the MRD group was decreased. This area is located at the frontal lobe and its anterior portion, as the region found in this study, is connected with most of the prefrontal cortex. ${ }^{28}$ Structural deficits in the supplementary motor area have been found in patients with depression with melancholic features and also explained their implicit learning deficits. ${ }^{29}$ Our finding of hypofrontality was in line with a recent study employing transcranial magnetic stimulation, ${ }^{14}$ in which the authors explicitly addressed the prefrontal cortex-related differences between MRD and non-MRD patient groups. They reported that the MRD group had a significantly greater resting motor threshold than the non-MRD group, suggesting that treatment resistance might be associated with prefrontal cortex hypoexcitability. ${ }^{14}$ Our study provides more direct evidence to support hypofrontality of patients with greater refractoriness. Major depression is considered a unique disorder based on DSM-IV criteria, yet a growing body of evidence indicates that the current criteria could have included a heterogeneous depressed population. Our finding of no correlation between past major depressive episodes and prefrontal cortex glucose metabolism suggests that the decreased glucose metabolism of the MRD group was not the result of repeated depression episodes. Besides, we also found that the length of the current episode was not a reliable feature of treatment refractoriness, because the mean length was greater in the non-MRD group, all of whom responded well to the prospective 6-week SSRI treatment. Thus, the remarkable features reflected in the difference in resting brain glucose metabolism between the MRD and non-MRD groups suggest that a history of refractoriness to antidepressants might be viewed as a trait feature. The heterogeneity of the population with major depressive disorder with respect to various degrees of treatment refractoriness might, at least in part, account for the inconsistencies in neuroimaging results previously reported for these patients. However, further study is warranted to identify whether the presence of decreased prefrontal cortex metabolism occurs early in the course of MRD.

Amygdala and association limbic regions are involved in depression, and many studies have highlighted the amygdala as a key region in the processing of negative emotion. ${ }^{10}$ However, we saw no significant difference in glucose metabolism in the amygdala between the three study groups. Although the glucose metabolism of the amygdala in the depression groups seemed to be higher than that in the controls (see Fig. DS2), no statistical significance was found in either voxel-based or region-of-interest analyses. Abnormal amygdala activity may not represent a persistent marker of depression common to all subtypes. For example, the results of a study directly investigating the amygdala using functional MRI with event-related experiments involving emotional faces revealed that only depressed patients with bipolar affective disorder, but not depressed patients with major depression or healthy controls, had elevated left amygdala activity in response to mild and neutral facial expressions in the 'sad' experiments. ${ }^{30}$ The study conclusion was that abnormally elevated left amygdala activity in response to mildly sad and neutral faces might be a depression-specific marker of bipolar disorder but not of major depression.

Hypometabolism of the left temporal gyrus was demonstrated in both depression groups. Analysis a posteriori revealed that this hypometabolism was not related to illness duration $(r=-0.084$, $P=0.643)$, past depressive episodes $(r=-0.084, P=0.659)$ or depression ratings $(r=-0.002, P=0.993)$. Resting state hypoactivity in the left temporal gyri has also been reported in patients with major depression receiving medication. ${ }^{31}$ The hypoactive temporal cortex might be considered to be an additional trait feature for depressed patients with major depressive disorder, although these mechanisms are as yet undetermined.

\section{Strengths and limitations}

The strength of our experimental design included stringent controls for many confounding factors that may have resulted in the inconsistencies of results of previous PET studies. Such factors include age, education, medication and presenting depressive severity (i.e. HRSD scores). ${ }^{23,32}$ However, some limitations should also be considered in the interpretation of our study. First, depression is heterogeneous, and this study took what we believe is the first step toward disentangling this unresolved issue. However, because of concerns of frontal metabolic changes due 
to electroconvulsive therapy (ECT), patients with extremely refractory depression were not our target population. The definitions of treatment-refractory or resistant depression varied widely across studies and an individual who responded poorly to antidepressant medications might eventually respond to other treatment options such as brain stimulation. Therefore, further studies are necessary to confirm whether the hypofrontality revealed in our MRD group characterises the pathophysiology of patients with refractory depression who have shown no response to ECT or to other antidepressant interventions that have the potential to treat MRD (e.g. newer generations of antidepressants or atypical antipsychotics). Notably, previous studies have reported that even before ECT, patients with MRD presented with significant hypometabolism in the bilateral frontal cortices. ${ }^{33}$ Besides, owing to ethical concerns and practical issues, our nonMRD group comprised patients who were newly diagnosed or relapsed and were not currently medicated. The optimal comparison group should be those who matched perfectly to the MRD group in terms of duration of illness and past mood episodes. However, even after adjusting for these confounders, our main results remained unchanged. Second, psychiatric comorbidities in depressed patients, such as anxiety, should be considered when interpreting our results. However, no significant difference in comorbidity was found between our depression groups. Third, a medication-free period before PET scanning and cognitive testing allowed us to minimise confounding effects from medications during the data interpretation, yet the potential long-term effects of past medication use cannot be completely overlooked. Finally, whether prefrontal cortical glucose metabolism is decreased early in the course of MRD cannot be answered from results of this study. To resolve this, as a next step we would like to scan a group of treatment-naïve individuals with first- episode major depressive disorder before the initiation of antidepressant treatment, and then follow their responses to sequential, adequate antidepressant trials.

\section{Study implications}

Patients with MRD, but not patients in the non-MRD and control groups, presented with pronounced prefrontal cortex glucose hypometabolism unrelated to the number of previous major depressive episodes. This hypometabolism explained why the MRD group performed worse in the attentional task. Prefrontal cortical rCMglu had low to moderate sensitivity and specificity as a biomarker for differentiating those with treatment resistance from the population with major depressive disorder. Our PET study, to our knowledge the first to be reported, confirmed that MRD can be biologically distinguished from non-MRD and control conditions. Early detection of cases resistant to medication could assist clinicians in selecting appropriate treatment strategies and thus prevent patients from repeated antidepressant treatment failures. Our results support the idea that MRD and non-MRD patients should be appreciated as two different subgroups of patients with major depressive disorder, both for clinical practice and for neuroimaging research purposes.

\section{Funding}

This study was sponsored by grants from the Taipei Veterans General Hospital (V100B-016, V102E3-006 V103E9-005 and V103E3-006), National Science Council (NSC 98-2752-B-010-001-PAE and NSC 98-2752-B-010-003-PAE) and Yen Tjing Ling Medical Foundation (Cl-100-6).

\section{Acknowledgements}

We would like to express our gratitude to all patients who kindly participated in this study and to all research assistants who assisted in the study.
Cheng-Ta Li, MD, PhD, Tung-Ping Su, MD, Department of Psychiatry, Taipei Veterans General Hospital, Taipei, Institute of Brain Science, and Division of Psychiatry, Faculty of Medicine, National Yang-Ming University, Taipei: Shyh-Jen Wang Wang, MD, Depart Mo Taiper, Pei-Chi Tu, MD, PhD, Department of Psychiatry, Taipei Veterans Gener Hospital, and Division of Psychiatry, Faculty of Medicine, National Yang-Ming University, Taipei; Jen-Chuen Hsieh, MD, PhD, Institute of Brain Science, National Yang-Ming University, Taipei, Integrated Brain Research Unit, Department of Medica Research and Education, Taipei Veterans General Hospital, Taipei, and Centre of Neuropsychiatric Research, National Health and Research Institute, ChuNan, Taiwan

Correspondence: Dr Jen-Chuen Hsieh, Integrated Brain Research Unit, Department of Medical Research and Education, Taipei Veterans General Hospital, No. 155, Sec. 2, Linong Street, Taipei 112, Taiwan. Email: jchsieh@ym.edu.tw; jchsieh@vghtpe.gov.tw

First received 16 Oct 2013, final revision 22 Apr 2014, accepted 29 Sep 2014

\section{References}

1 Warden D, Rush AJ, Trivedi MH, Fava M, Wisniewski SR. The STAR*D Project results: a comprehensive review of findings. Curr Psychiatr Rep 2007; 9: 449-59.

2 Li CT, Lin CP, Chou KH, Chen IY, Hsieh JC, Wu CL, et al. Structural and cognitive deficits in remitting and non-remitting recurrent depression: a voxel-based morphometric study. Neuroimage 2010; 50: 347-56.

3 Trivedi $\mathrm{MH}$, Hollander E, Nutt D, Blier P. Clinical evidence and potential neurobiological underpinnings of unresolved symptoms of depression. J Clin Psychiatry 2008; 69: 246-58.

4 Berlim MT, Turecki G. What is the meaning of treatment resistant/refractory major depression (TRD)? A systematic review of current randomized trials. Eur Neuropsychopharmacol 2007; 17: 696-707.

5 Little A. Treatment-resistant depression. Am Fam Physician 2009; 80: 167-72.

6 Souery D, Amsterdam J, de Montigny C, Lecrubier Y, Montgomery S, Lipp O, et al. Treatment resistant depression: methodological overview and operational criteria. Eur Neuropsychopharmacol 1999; 9: 83-91.

7 Thase ME, Friedman ES, Biggs MM, Wisniewski SR, Trivedi MH, Luther JF, et al. Cognitive therapy versus medication in augmentation and switch strategies as second-step treatments: a STAR*D report. Am J Psychiatry 2007; 164: 739-52.

8 Price JL, Drevets WC. Neurocircuitry of mood disorders. Neuropsychopharmacology 2010; 35: 192-216.

9 Hoflich A, Baldinger $P$, Savli M, Lanzenberger R, Kasper S. Imaging treatment effects in depression. Rev Neurosci 2012; 23: 227-52.

10 Drevets WC, Price JL, Furey ML. Brain structural and functional abnormalities in mood disorders: implications for neurocircuitry models of depression. Brain Struct Funct 2008; 213: 93-118.

11 Johnstone T, van Reekum CM, Urry HL, Kalin NH, Davidson RJ. Failure to regulate: counterproductive recruitment of top-down prefrontal-subcortical circuitry in major depression. J Neurosci 2007; 27: 8877-84.

12 Paus T, Barrett J. Transcranial magnetic stimulation (TMS) of the human frontal cortex: implications for repetitive TMS treatment of depression. J Psychiatry Neurosci 2004; 29: 268-79.

13 Kimbrell TA, Ketter TA, George MS, Little JT, Benson BE, Willis MW, et al. Regional cerebral glucose utilization in patients with a range of severities of unipolar depression. Biol Psychiatry 2002; 51: 237-52.

14 Levinson AJ, Fitzgerald PB, Favalli G, Blumberger DM, Daigle M, Daskalakis ZJ. Evidence of cortical inhibitory deficits in major depressive disorder. Biol Psychiatry. 2010; 67: 458-64.

15 Kennedy SH, Evans KR, Kruger S, Mayberg HS, Meyer JH, McCann S, et al. Changes in regional brain glucose metabolism measured with positron emission tomography after paroxetine treatment of major depression. Am J Psychiatry 2001; 158: 899-905.

16 Sheehan DV, Lecrubier $\mathrm{Y}$, Sheehan $\mathrm{KH}$, Amorim $\mathrm{P}$, Janavs J, Weiller $\mathrm{E}$, et al. The Mini-International Neuropsychiatric Interview (M.I.N.I.): the development and validation of a structured diagnostic psychiatric interview for DSM-IV and ICD-10. J Clin Psychiatry 1998; 59 (suppl 20): 22-33.

17 American Psychiatric Association. Diagnostic and Statistical Manual of Mental Disorders (4th edn) (DSM-IV). APA, 1994.

18 Hamilton M. A rating scale for depression. J Neurol Neurosurg Psychiatry 1960; 23: 56-62.

19 Young RC, Biggs JT, Ziegler VE, Meyer DA. A rating scale for mania: reliability, validity and sensitivity. Br J Psychiatry 1978; 133: 429-35. 
20 Gispert JD, Pascau J, Reig S, Martinez-Lazaro R, Molina V, Garcia-Barreno P et al. Influence of the normalization template on the outcome of statistical parametric mapping of PET scans. Neuroimage 2003; 19: 601-12.

21 Hirvonen J, Goodwin RS, Li CT, Terry GE, Zoghbi SS, Morse C, et al. Reversible and regionally selective downregulation of brain cannabinoid CB1 receptors in chronic daily cannabis smokers. Mol Psychiatry 2012; 17: 642-9.

22 Tzourio-Mazoyer N, Landeau B, Papathanassiou D, Crivello F, Etard O, Delcroix N, et al. Automated anatomical labeling of activations in SPM using a macroscopic anatomical parcellation of the MNI MRI single-subject brain. Neuroimage 2002; 15: 273-89.

23 Fitzgerald PB, Oxley TJ, Laird AR, Kulkarni J, Egan GF, Daskalakis ZJ. An analysis of functional neuroimaging studies of dorsolateral prefrontal cortical activity in depression. Psychiatr Res 2006; 148: 33-45.

24 Soreide $\mathrm{K}$, Korner H, Soreide JA. Diagnostic accuracy and receiver-operating characteristics curve analysis in surgical research and decision making. Ann Surg 2011; 253: 27-34.

25 Brody AL, Saxena S, Stoessel P, Gillies LA, Fairbanks LA, Alborzian S, et al. Regional brain metabolic changes in patients with major depression treated with either paroxetine or interpersonal therapy: preliminary findings. Arch Gen Psychiatry 2001; 58: 631-40.

26 Holthoff VA, Beuthien-Baumann B, Zundorf G, Triemer A, Ludecke S, Winiecki $P$, et al. Changes in brain metabolism associated with remission in unipolar major depression. Acta Psychiatr Scand 2004; 110: 184-94.
27 Teneback CC, Nahas Z, Speer AM, Molloy M, Stallings LE, Spicer KM, et al. Changes in prefrontal cortex and paralimbic activity in depression following two weeks of daily left prefrontal TMS. J Neuropsychiatr Clin Neurosci 1999; 11: 426-35.

28 Zhang S, Ide JS, Li CS. Resting-state functional connectivity of the medial superior frontal cortex. Cereb Cortex 2012; 22: 99-111.

29 Exner C, Lange C, Irle E. Impaired implicit learning and reduced presupplementary motor cortex size in early-onset major depression with melancholic features. J Affect Disord 2009; 119: 156-62.

30 Almeida JR, Versace A, Hassel S, Kupfer DJ, Phillips ML. Elevated amygdala activity to sad facial expressions: a state marker of bipolar but not unipolar depression. Biol Psychiatry 2010; 67: 414-21.

31 Hosokawa T, Momose T, Kasai K. Brain glucose metabolism difference between bipolar and unipolar mood disorders in depressed and euthymic states. Progr Neuropsychopharmacol Biol Psychiatry 2009; 33: 243-50.

32 Videbech P. PET measurements of brain glucose metabolism and blood flow in major depressive disorder: a critical review. Acta Psychiatr Scand 2000; 101: 11-20.

33 Suwa T, Namiki C, Takaya S, Oshita A, Ishizu K, Fukuyama $H$, et al. Corticolimbic balance shift of regional glucose metabolism in depressed patients treated with ECT. J Affect Disord 2012; 136: 1039-46.

AD EXTRA

\title{
reflection
}

\section{On Beyond Freedom and Dignity by B. F. Skinner}

\author{
Lynne M. Drummond
}

In 1971, the Western world was in the midst of the Cold War and debates raged about how we should act to ensure our long-term security and safety. In the same year, Burrhus Frederic Skinner published his highly controversial book, Beyond Freedom and Dignity. Already renowned as a behaviourist, author, social philosopher, inventor and professor of psychology at Harvard University, he set out to develop the ideas of how we could solve the world's growing problems. The controversial element was that, unlike conventional philosophical thought, Skinner did not consider man as fundamentally different from animals. Strongly influenced by Darwin's idea of natural selection, he believed that man, just like other animals, performs behaviours which are reinforcing or avoid aversive consequences. Unlike previous philosophers who believed that man has internal drives to do good, be heroic or make a choice to perform antisocial acts, Skinner believed these were outside our internal control and produced by environmental reinforcers. Applying strict scientific method, he wished to explore these complex reinforcers. He believed that social policy should encourage and reinforce desirable behaviours for the benefit of society at large. These views were not only against conventional religious and philosophical thought, but also against the continuous mantra that individuals should be free and able to make up their own minds about their actions without interference. Many critics appeared confused about what constitutes reinforcement and Skinner was often portrayed as advocating an overly punitive system of control. In fact, he advocated the use of positive reinforcers and some negative reinforcers. Positive reinforcers comprise tangible benefits (wealth, physical possessions) as well as social approval. Negative reinforcers are consequences which can be avoided by acting in a certain way. Skinner was not a believer in excessive use of punishment but felt people should be 'shaped' to behave better. No human being was truly free but made choices based on multiple, complex, and small environmental reinforcers, partially determined by our genetic structure. Dignity was an erroneous idea based on the concept of the 'inner man' being responsible for worthy acts as well as misdeeds: 'Almost all major problems involve human behavior, and they cannot be solved by physical and biological technology alone. What is needed is a technology of human behavior'.

The relevance of this work over 40 years later is striking. Today, we have the threats of environmental disasters, climate change, multiple wars and a population of over 7 billion. In the Western world we stand firm to the mantra that we must be free and must avoid allowing the politicians to develop a 'nanny state'. Despite this, when efforts have been made to alter the reinforcers for detrimental human behaviour, such as increasing social isolation of smokers, beneficial effects have been seen. All thinking individuals will resist any extreme introduction of state control and lack of freedom, but maybe the reinforcers are currently stacked to encourage antisocial rather than socially beneficial behaviours. Controversial trials of paying individuals to develop healthier lifestyles and lose weight have been implemented. Indeed, in cognitive-behavioural therapy we are now moving increasingly away from more cognitive models of treatment and back to examining the behavioural routes of many psychiatric syndromes. Perhaps it is time to revisit this thought-provoking and innovative book. 